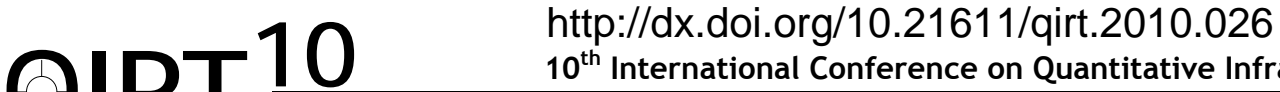 \\ $10^{\text {th }}$ International Conference on Quantitative InfraRed Thermography \\ July 27-30, 2010, Québec (Canada)
}

\section{Full Field Experimental stress/strain analysis by Thermographic stress analyser for fatigue crack detection during HCF Testing. Examples in Automotive and Aircraft industry.}

by P. Bremond*

*FLIR ATS, 19 Bd G. Bidault Croissy Beaubourg, France, pierre.bremond@flir@fr

\begin{abstract}
Thermographic Stress Analysis (TheSA) is a well established as a full field non contact technique to measure stress on material surface. This technique uses thermo-elastic coupling and mechanical heat dissipation in order to measure stress or dissipated energy by infrared camera and real time signal processing embedded in optical head. The principle is to detect thanks to a reference signal the dynamic temperature change synchronized with external mechanical energy (load) applied to specimen or the real structure. Under adiabatic assumption which depends on material properties, load frequency and stress concentration, the map of the sum of principle stresses and dissipated energy can be measured in seconds.
\end{abstract}

Compared to contact techniques (cracks gauges) or NDT technique (liquid penetrent, C scan view), full field measurement techniques enable to detect and quantify heterogeneities in stress and dissipated energy without stopping fatigue testing. "Heterogeneities" are therefore being regarded as the common purpose of these full field techniques.

As it is predicted by the fracture mechanics, propagating fatigue crack creates stress concentration with high level of stresses. This makes crack detection more easily detectable by Thermographic Stress Analyzer. Moreover local plasticity on tip of crack can be also detected by TheSA thanks to dissipated energy measurement.

The aim of this paper is to introduce some examples of use of TheSA during High Cycle Fatigue (HFC) for detection appearance of fatigue crack on metallic components in automotive and aircraft industry. The framework of stress and dissipated energy will be reminded before to evaluate the specifications of infrared camera required for such applications.

Some examples in each sector will be presented and finally a minimum crack detection length will be established thanks to laboratory test.

Keywords: Thermo-elasticity, TheSA, Digital image correlation, HCF, Fatigue, Dissipated energy, full field imaging, crack detection, wireless Lock in thermography, Long distance

\section{Introduction}

In the Mechanical Engineering and Non destructive Testing fields the last decades have seen a fast development of full field measurements. The improvement in image processing with microcomputers and the advances in camera technology have made non contact measurement techniques such as Digital Image Correlation, Interferometry Speckle and Thermographic Stress Analysis (TheSA) to become more and more popular in the experimental mechanics and NDT communities.

Full field measurement techniques enable to detect and to quantify heterogeneities in displacement, strain, temperature and stress fields. "Heterogeneities" are therefore being regarded as the common purpose of these techniques.

Those heterogeneities are already used at different stages for instance:

To discover some local phenomena which are not accounted by the global behavior.

To perform unbiased tests, for instance by detecting some unexpected heterogeneities due to the specimen shape or illcontrolled boundary conditions.

To verify the validity of some assumptions under which some models describing the mechanical response of structures are conducted.

To compare experiment result to their numerical counterparts.

To extract simultaneously an important number of constitutive parameters on a single specimen. 
High Cycle Fatigue testing is one of the mechanical testing in which heterogeneities are looked for. Specimen or full scale structures are undergoing long time dynamic loading until appearance the first crack. The test is usually stopped periodically in order to perform a Non Destructive Testing technique to detect microscopic crack.

Thermographic Stress Analyzer is able to detect such heterogeneity of stress map or dissipated energy due to local high stress concentration around crack tip. Some example in Automotive, Civil engineering and Aircraft industry will be presented.

\section{Mechanical and Thermo-mechanical framework}

Material or component undergoing mechanical testing transforms the mechanical energy applied (load) into strain or stored it as permanent deformation. The relationship between the applied load and the strain are described by constitutive equation. This equation links the strain tensor to stress tensor (and their time derivatives).

$$
\sigma=f(\varepsilon, \& T)
$$

Thermo-mechanical framework allows describing the same test under the thermodynamics consideration. By combining the first and second principles of thermodynamics, the local equation of heat diffusion describes the energy exchange between mechanical energy and heat. It appears that the global heat generated by mechanical load can be separated into thermo-elastic energy and dissipated energy. The first energy measurement can easy be used to measure directly local stress by knowing of only 3 material properties (density, specific heat and linear expansion). One can note that constants of constitutive equation are not required. By complementary image processing, dissipated energy is separately measured. This value is usually not used for absolute value but allows detecting some local heterogeneity of plastic energy production and consequently fatigue limit and future crack.

$$
\rho \cdot C \cdot \mathcal{L}_{k} \cdot \Delta_{x, y} T=W_{\text {the }}^{\&}+d_{1}+r_{e}
$$

\section{Full field measurement techniques.}

Digital Image processing is computer based process to obtain a 2-D full field information by recording deformation and motion of speckle patterns on a specimen surface before and after deformation of the body. It takes advantage of the fact that applied stresses change both the thickness and optical properties of materials, to determine displacement. Then strains are obtained from the 2D displacement pattern.

The equipment is less demanding as optically-ordinary incoherent light is sufficient and no need for optical components. It requires one Hi resolution camera for 2D displacement and for 3D displacement, 2 cameras. A computer and frame grabbing circuit card to digitize the output.

The result is a differential image of strain. Local constitutive equation is required for stress calculation.

The long distance detection is limited by the spatial resolution of camera.

Thermographic Stress Analysis is Digital thermal image processing which provides in real time the temperature change due to continuous dynamic mechanical load. The processing is a Fourier Transform synchronized on the load signal. The TheSA can provide both stress and dissipated energy patterns at the tip of crack. To do so no constitutive equation is required. The frequency spectrum of mechanical load which can be processed is $3 \mathrm{~Hz}$ to $10 \mathrm{kHz}$ independently to the camera frame. The main advantages of TheSA for crack detection are:

High stress concentration zone around the crack tip is larger than tip size itself

The signal is maximum at crack tip

Lock in thermography has sub pixel detection

Dissipated energy can predict crack or detect sub surface crack

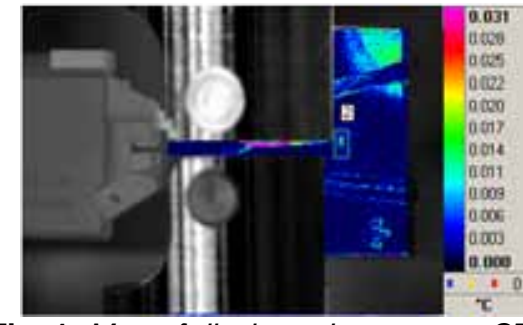

Fig. 1. Map of dissipated energy on CT specimen

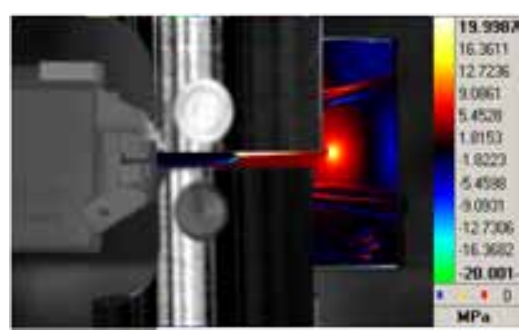

Fig. 2. Map of stress on CT specimen 
A method to extract the load signal from the thermal image will be introduced. It allows using TheSA without any contact with component and structures undergoing $\mathrm{HC}$ fatigue testing. A periodic inspection can then be performed periodically without stopping the fatigue test.

\section{Example of crack detection during HCF testing}

The following example shows the result obtained during a High Cycle fatigue testing on riveted aluminum plates performed at the same level of stress. The two images shown are taken after 190000 cycles. Visible cracks are already observed in two fasteners on the top left while no crack is visible in the two fasteners on the right.

The results are obtained by real time processing with TheSA system. The stress image on the left confirms stress concentration at the tip crack and doesn't show any crack in the third column.

The right image is dissipated energy map obtained during the same cycle. Dissipated energy can be observed around fasteners in column 1 and 2. Nevertheless the column 3 and 4 shows also local dissipated energy.

After removal a crack between the two plates was discovered.
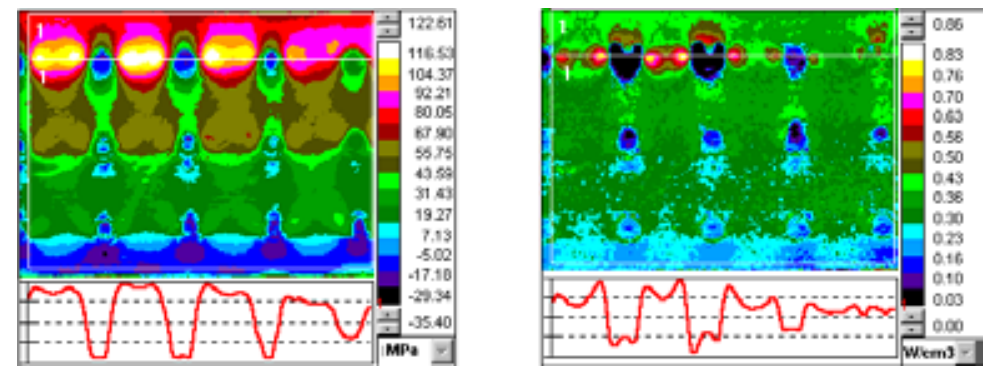

Fig. 3. Map of dissipated energy and dissipated energy recorded after 90000 cycles on riveted aluminum plates

\section{Conclusion}

Thermographic Stress Analysis is a powerful non contact full field technique to detect crack during HCF testing of metallic components or structures. Lock in detection allows sub pixel spatial resolution.

It measures in real time the high stress concentration around the crack tip. Dissipated energy measurement can also in some cases give good result for subsurface crack detection in thin material. presentation.

Some cases of Civil engineering (bridge) and automotive industry (engine) will be presented during the oral

\section{REFERENCES}

[1] Brémond P.: “Développement d'une instrumentation infrarouge pour la mesure des contraintes - Application aux fissures de fatigue". Univ. Marseille. 1982.

[2] Mann J.Y.: "Aircraft fatigue with particular emphasis on Australian operations and research", $12^{\text {th }}$ ICAF symp. Toulouse. 1983.

[3] Chrysochoos A.: "Stored energy measurements for behavior laws by thermomechanical analysis", ITCAM Grenoble. 1988.

[4] Luong M.P.: "Infrared Thermographic scanning of fatigue in metals". $12^{\text {th }}$ International conference on SMIRT. 1993.

[5] Brémond P., P otet P.: "Démodulation synchrone des images thermiques. Application à l'analyse expérimentale des structures".SFT.1994.

[6] Luong M. P.: "F atigue limit evaluation of metals using an infrared technique". Mech. of Mat. 1997.

[7] Peyroux R., Chrysochoos A., Licht C., Löbel M.: "Thermo mechanical couplings and pseudo elasticity of shape memory alloys". Int. J. Engng Sci. 1998.

[8] Wang H., Dinwiddie R.B.: "Application of High Speed IR imaging During Mechanical Fatigue Tests". Thermosense XXII. 2000. 